\title{
EL TURISMO DE INTERIOR EN EL DESARROLLO SOCIOECONÓMICO DE LAS COMARCAS FORESTALES DE LA COMUNIDAD VALENCIANA ${ }^{1}$
}

\author{
Cristina Montiel Molina \\ Departamento de Análisis Geográfico Regional y Geografía Física \\ Universidad Complutense de Madrid
}

\section{RESUMEN}

El objetivo de este artículo es demostrar la interacción que debe existir entre la política de desarrollo del turismo y la recuperación de los espacios forestales. Si las actividades agrícolas, ganaderas y forestales contribuyen al mantenimiento de los ecosistemas naturales, que son la base de la demanda turística en las comarcas interiores, la planificación del turismo rural no puede realizarse al margen de dichas actividades tradicionales, a las que se debe garantizar su viabilidad y permanencia para asegurar el éxito de la oferta turística. En este planteamiento convergen los principios de multifuncionalidad y sostenibilidad de la gestión forestal y del turismo.

Desde estas premisas, se realiza una propuesta de ordenación de los espacios forestales orientada a su utilización con fines recreativos y turísticos e integrada en las políticas de desarrollo rural. En segundo lugar, se define posibles vías de instrumentación de las políticas de revalorización paisajística y de uso público de los montes valencianos.

Palabras clave: Turismo rural, monte mediterráneo, usos turístico-recreativos, desarrollo sostenible, multifuncionalidad, Comunidad Valenciana.

\begin{abstract}
This paper aims to demonstrate the interaction that should exist between tourist development strategies and the forestland management. If the agricultural, cattle and forestry activities contribute to the conservation of natural ecosystems, which are the basis of the tourist demand in the inner regions, then the planning of ecotourism should not be developed without those traditional activities. On the contrary, the planning of ecotourism should ensure the viability and the permanence of those activities in order to guarantee the
\end{abstract}

1 Este artículo es el texto actualizado de la ponencia desarrollada por la autora en el Congreso «El monte mediterráneo y los ciclos vitales asociados», organizado por la Consellería de Medio Ambiente de la Generalitat Valenciana en Valencia, los días 27 al 30 de noviembre de 2000. 
sucess of the tourist supply. This is the way to integrate the principles of multifonctionality and sustainable forest management with the sustainability of tourism.

From this premise, a landforest planning for leisure and tourist uses is proposed within the rural development policies. Next, some posible implementation instruments for landscape valorization strategies and for the public use of Valencia's forestlands mesures are defined.

Key words: Eco-tourism, Mediterranean forests, leisure and tourist uses, sustainable development, multifonctionality, Valencia Autonomous Comunity.

\section{Introducción}

La mayor parte de los estudios realizados durante los últimos quince años sobre el turismo rural y sobre los espacios de ocio de elevada calidad ambiental ignoran la función que desempeñan los ecosistemas forestales en el desarrollo socioeconómico de las comarcas afectadas. Frente a la atención dedicada a los alojamientos, actividades en el medio rural, infraestructuras y rasgos sociodemográficos de las áreas rurales, apenas se realizan alusiones a los espacios forestales, siquiera como marco paisajístico y ambiental de la oferta turística.

Además, el desarrollo del turismo rural en la Comunidad Valenciana parte del condicionante negativo que supone la valoración principal de la región, desde el punto de vista turístico, en relación al modelo dominante de sol y playa. Al igual que en el resto de las regiones mediterráneas con una franja litoral desarrollada, las áreas del interior despiertan relativamente escaso interés frente a la costa, a pesar de la calidad de sus recursos ambientales y paisajísticos.

Por otra parte, el turismo ha sido generalmente considerado un factor de riesgo desde la perspectiva forestal por contribuir a desencadenar o agravar procesos de degradación en ecosistemas frágiles y vulnerables. La propia Estrategia Forestal Española continúa enfatizando los posibles impactos ambientales negativos de las funciones recreativas y turísticas e insiste en la necesidad de establecer límites y normas precisas. En cambio no hace alusión alguna a los efectos positivos que estas funciones podrían igualmente ejercer sobre la recuperación y reutilización de los montes. En este sentido, los planteamientos de la Estrategia Forestal Española continúan siendo parciales, ya que no integran los montes, desde un punto de vista económico y territorial, en las políticas de desarrollo rural ni en las políticas turísticas: "En términos generales, la planificación y gestión de zonas forestales para el uso recreativo llevará consigo un tipo de actuación dirigido básicamente hacia la elección de zonas específicas y su adecuación a los objetivos con el establecimiento de accesos, infraestructuras y servicios que optimicen los usos propuestos, la regulación, dirección y control de los visitantes y la oferta de servicios de información y, en determinados montes, de educación ambiental» (Ministerio de Medio Ambiente, 2000: 66-67).

Frente a estas posturas, en los últimos cinco años se viene observando, sin embargo, una progresiva valoración positiva de las actividades turístico-recreativas en las comarcas forestales del interior de las regiones mediterráneas, integrada en una visión global de desarrollo rural (Cardells, 1997). Entre otros efectos, se subraya la contribución del turismo a la recuperación demográfica y revitalización de las comarcas rurales interiores; la contribución a la educación ambiental y a la formación de una conciencia social que valore la necesidad de 
proteger el medioambiente; y la recuperación de los montes a través de su reutilización. El uso público de los ecosistemas forestales puede ser, por tanto, una de las vías de recuperación de unos espacios que desde mediados de siglo se han venido caracterizando por el abandono demográfico y socioeconómico y por la infragestión.

\section{La multifuncionalidad del monte mediterráneo}

Los montes mediterráneos son ecosistemas naturales y culturales en los cuales la antropización secular ha dejado huellas paisajísticas que forman ya parte de su idiosincrasia. El paisaje forestal mediterráneo cuenta con una fortísima componente humana de carácter histórico, de manera que su dinámica no es sólo natural, sino también social y cultural (Montiel, 1996 y 1999). La presencia humana en el monte mediterráneo, a pesar de los efectos negativos que también ha provocado, ha sido fundamental para garantizar la protección y restauración de estos ecosistemas. Por ello, el proceso de abandono de usos y aprovechamientos tradicionales en las áreas forestales, unido al éxodo rural, es una de las principales explicaciones de la crisis y problemas que afectan en la actualidad a los montes mediterráneos ${ }^{2}$.

La evolución socioeconómica que han experimentado los montes mediterráneos a lo largo del siglo XX se ha caracterizado por la transición de los aprovechamientos vinculados al sector primario durante la primera mitad de la centuria a la terciarización de usos y funciones tras el proceso de abandono de aprovechamientos tradicionales y éxodo rural que se inicia en los años sesenta. La subexplotación de los recursos forestales, unida a la transformación de los usos del monte que se ha producido como consecuencia de la generalización del modo de vida urbano y el abandono de los aprovechamientos tradicionales en los espacios rurales, han conducido a la crisis de las comarcas forestales del interior.

La valoración que durante las últimas dos décadas ha recibido el monte mediterráneo está fundamentalmente relacionada con su significado paisajístico, con la conservación de la biodiversidad y con la demanda de espacios de ocio al aire libre. Los nuevos usuarios de estos espacios ya no proceden del ámbito local. De manera que el valor económico del monte no ha desaparecido, pero sí ha cambiado de naturaleza: frente a los rendimientos tradicionales, de carácter local y material, los beneficios actuales son indirectos y están deslocalizados (figura 1).

A tenor de la evolución reciente, resulta evidente que el uso público recreativo, educacional y turístico de los ecosistemas forestales mediterráneos constituye una de las principales funciones de los mismos (Montiel, 1994), integrada además en el nuevo modelo de desarrollo rural impulsado desde la Unión Europea ${ }^{3}$. Recordemos que la Estrategia Forestal para la Unión Europea aprobada el 15 de diciembre de 1998 pone de relieve la importancia del papel plurifuncional de los bosques y de la explotación sostenible del sector forestal. Recogiendo y desarrollando estos principios, la Estrategia Forestal Española se hace eco del proceso de terciarización que afecta a los montes españoles y reconoce la prioridad que a menudo adquiere la función recreativa sobre cualquier otro tipo de función productiva tradicional.

2 Declaración Final de la Conferencia Internacional sobre la Conservación y el Uso Sostenible del Monte Mediterráneo, Málaga, octubre 1998 (Ministerio de Medio Ambiente, 2000: 236-238).

3 Reglamento (CE) n 1257/1999 del Consejo de 17 de mayo de 1999 sobre la ayuda al desarrollo rural a cargo del FEOGA. 
En la Comunidad Valenciana, concretamente, se estima en 5.789.180 el número de visitas anuales que, con dicha finalidad, registran los espacios forestales. El problema radica en la dificultad de valorar estos usos terciarios de los montes, por no tener la mayoría de ellos precios de mercado (Cardells, 2001). Se plantea, por tanto, la necesidad de la planificación e intervención territorial para regular la dinámica de las externalidades positivas producidas por los ecosistemas forestales, ante las limitaciones evidenciadas por los mecanismos de mercado.

Actualmente, existe una creciente demanda social hacia la naturaleza que todavía no se ha resuelto con actitudes y modelos de gestión nuevos y satisfactorios, lo cual entraña un grave riesgo de progresiva pérdida de interrelación con el territorio. Si los ecosistemas forestales se abandonaron por su falta de rentabilidad, la recuperación de los mismos comporta necesariamente una justificación económica. Y para ello debe producirse una internalización de las externalidades positivas (sociales y paisajísticas) que generan los montes (Merlo y Rojas, 2000). Partiendo del hecho de que el abandono de los montes por su baja rentabilidad causa unos perjuicios globales mucho mayores que el pequeño coste que supone el mantenimiento de su gestión, se trata de internalizar las externalidades positivas generadas por los montes mediterráneos involucrando particularmente a la actividad turística. Se trata, en definitiva, de establecer una política activa de ecotasas que cuenta con precedentes en algunos países centroeuropeos y que, combinada con una posible vía de contratos de conservación paisajística, permitiría llevar a cabo una gestión forestal y paisajística eficaz (Rojas, 1999).

La gestión del monte mediterráneo en la actualidad obliga, en consecuencia, a superar modelos interpretativos del pasado y plantea el reto de definir un nuevo modelo de silvicultura cuyo objetivo principal, en respuesta a las nuevas demandas, sea la conservación de la biodiversidad y el mantenimiento del paisaje para favorecer la acogida al público de procedencia urbana y la apertura al turismo rural (Lacaze, 2000). La gestión del monte mediterráneo debe priorizar los fines protectores y sociales, atendiendo las demandas actuales con fórmulas originales que, partiendo de la especificidad de la región, favorezcan tanto la recuperación de las prácticas agrosilvopastorales tradicionales como la consolidación de los nuevos usos. Desde este punto de vista, el agroturismo o turismo rural constituye una interesante alternativa para lograr el desarrollo sostenible en las áreas interiores de la región mediterránea española, y concretamente en la Comunidad Valenciana. Además de la indiscutible revalorización patrimonial que estas actividades han inducido en las tierras interiores valencianas, la necesidad de recursos técnicos y humanos para mostrar, conservar y mejorar los ecosistemas que sirven de soporte al agroturismo pueden favorecer la recuperación demográfica de comarcas que han sufrido desde los años sesenta un intenso éxodo rural (Reina, 1992).

El turismo en comarcas forestales, planteado en el marco general del desarrollo local, debe favorecer, en consecuencia, la revitalización del medio rural. Pero también debe garantizar una mejor protección de los ecosistemas forestales mediante su gestión y mantenimiento, ya que el paisaje forestal es fundamental para asegurar el atractivo turístico. Evidentemente, la Administración no puede transformarse en agente empresarial que gestione la actividad turística con fines lucrativos en las comarcas del interior. Su función es la de favorecer el descubrimiento del patrimonio forestal por parte de la población y agentes locales y regionales, estableciendo las pautas precisas para la rentabilización de un uso turístico que no ponga en peligro los valores naturales y culturales del monte sino que, por el contrario, contribuya a su conservación. 


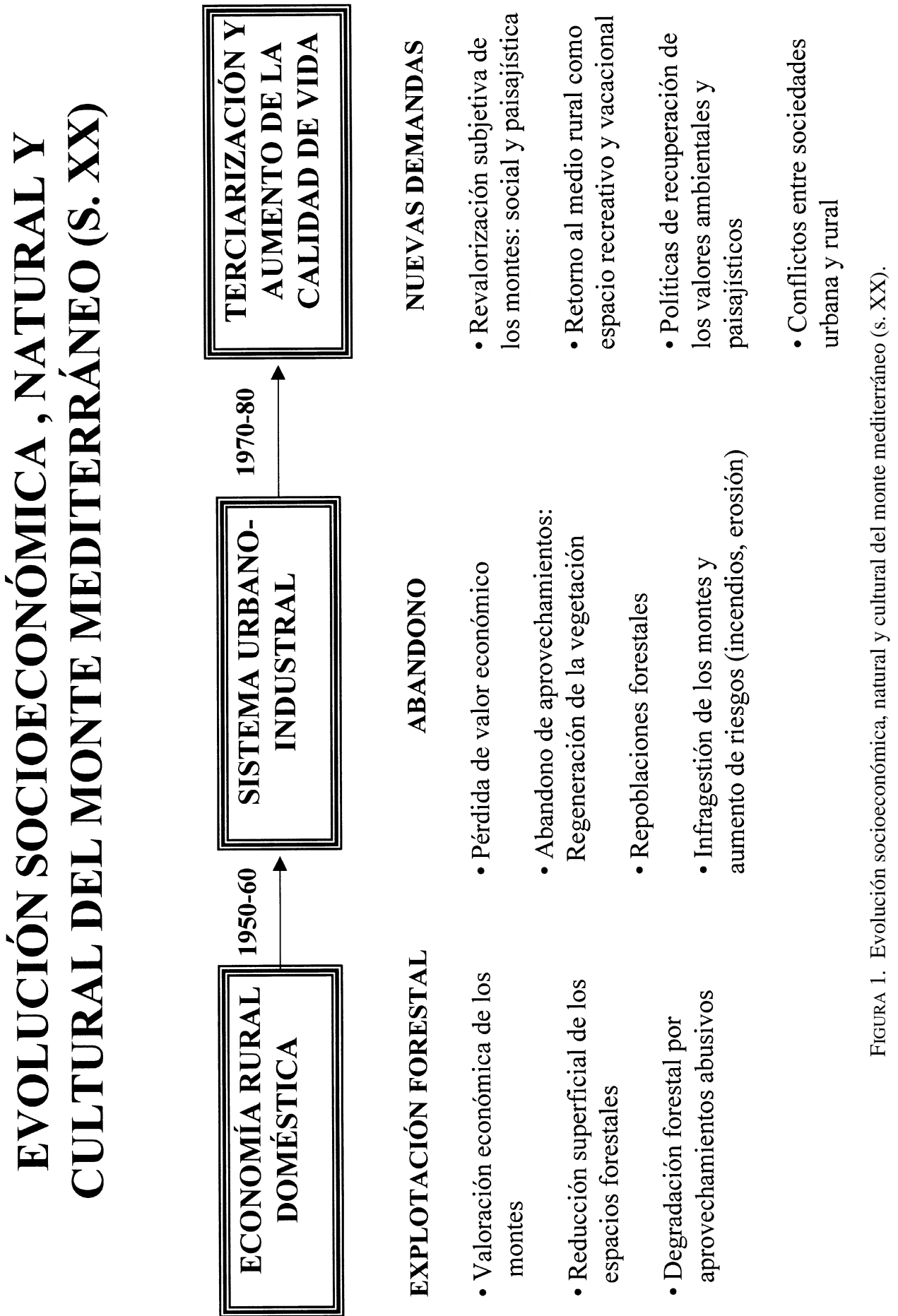

Investigaciones Geográficas, $n^{\circ} 31$ (2003) 
En suma, se trata de potenciar los rendimientos terciarios del monte y favorecer la multifuncionalidad de los ecosistemas forestales mediterráneos integrando en su dinámica las nuevas demandas y los aprovechamientos tradicionales que puedan contribuir a la recuperación de estos espacios. Los usos turísticos ofrecen, en este sentido, una magnífica oportunidad para el redescubrimiento y la «reapropiación», por parte de la sociedad, de los valores naturales y culturales del monte mediterráneo tras décadas de abandono, desuso y desconocimiento (AIFM, 2002).

En este sentido, y partiendo de los objetivos definidos por la Estrategia Forestal Española en relación al fomento de los usos terciarios del monte, la metodología de trabajo planteada se articula en torno a dos fases. En primer lugar, la evaluación del patrimonio existente ${ }^{4}$ : potencialidades y recursos turísticos de las comarcas forestales. En segundo lugar, la planificación de los usos públicos: incremento y diversificación de la oferta; e integración de las funciones turístico-recreativas de los montes en las políticas de desarrollo rural.

\section{Potencialidades y recursos turísticos de los ecosistemas forestales mediterráneos}

En las reuniones internacionales y documentos elaborados durante los últimos ocho años sobre la relación existente entre turismo y medioambiente se han realizado diferentes alusiones a la necesidad de integrar los objetivos de conservación de la naturaleza y el paisaje en las políticas de turismo y ocio, en aras de la sostenibilidad ecológica. La Estrategia Paneuropea sobre Diversidad Biológica y Paisajística, suscrita por 55 países europeos en Sofía en 1995 establecía una acción coordinada en la materia. Y en la misma línea se pronunciaba la Declaración acerca de las bases futuras de las actividades turísticas según el principio de sostenibilidad, adoptada en la Conferencia Internacional sobre Biodiversidad y Turismo que se celebró en Berlín en marzo de 1997: «El turismo deberá contribuir a la conservación, protección y restauración de los ecosistemas terrestres (...) La protección del medioambiente deberá formar parte del proceso de desarrollo turístico» (Consejo de Europa, 1997). Igualmente, la Declaración sobre Ecoturismo de Quebec de 2002, recomienda al sector privado la cooperación con las organizaciones gubernamentales y no gubernamentales que se ocupan de los espacios naturales protegidos y de la conservación de la biodiversidad, para garantizar que las actuaciones del ecoturismo se llevan a cabo de acuerdo con los planes de gestión y demás regulaciones existentes en dichos espacios, con objeto de minimizar los impactos negativos, al tiempo que se mejora la experiencia turística y ésta contribuye financieramente a la conservación de los recursos naturales.

Sin, embargo, los términos de relación entre turismo y medioambiente se han reducido, a menudo, a unas formulaciones vagas y generales. Han sido frecuentes, por ejemplo, las alusiones a la compatibilización, respeto, complemento, favorecimiento, y no tanto las implicaciones y los compromisos concretos que expresan otro tipo de conceptos, como la contractualización o la responsabilidad compartida. En general, se reconoce y subraya la importancia de los valores ambientales y paisajísticos para el desarrollo sostenible del turismo, pero no se diseñan en paralelo líneas políticas e instrumentos de gestión orientados a estos fines. De manera que tales propósitos quedan en la mera declaración de principios o intenciones.

4 «Es necesario conocer la oferta existente en la actualidad, el potencial de las distintas zonas, y su capacidad de atracción para los diferentes usos» (Ministerio de Medio Ambiente, 2000: 139). 
La relación entre actividades turísticas y ecosistemas forestales se ha reducido, en consecuencia, a la coexistencia de dos realidades o sectores independientes. Y en ello ha influido considerablemente la actitud preventiva del sector forestal hacia el uso público de los montes. Hasta fechas recientes ha primado una valoración generalista y simplista de signo negativo que atribuía a los usos turístico-recreativos toda una serie de impactos que habían terminado convirtiéndose en un listado de lugares comunes que se aplicaban indiscriminadamente: «Las instalaciones recreativas son muy necesarias. Las ciudades y pueblos demandan cada vez más espacios naturales para el solaz y disfrute. Pero si la entrada no se regula, se acaba produciendo una anarquía en el uso del monte, con grave peligro de deterioro. Por ello se debe dar la solución de adecuar núcleos de recreo, bien acondicionados, con nulo peligro de incendios y que absorban plenamente la demanda social»5.

Evidentemente, identificar la compleja función social de los montes mediterráneos con la mera adecuación de zonas recreativas es un error porque excluye el verdadero valor turístico y paisajístico de estos ecosistemas y la demanda social real, conduciendo además a entender el uso público de los montes en términos negativos de impactos ecológicos por prácticas intensivas. Si bien es cierto que las actividades turístico-recreativas, incorrectamente planteadas o realizadas pueden generar efectos perniciosos en los montes - al igual que ocurre con el resto de las actividades y aprovechamientos tradicionales-, no es menos cierto que, en el marco de una planificación y gestión ordenada, sus efectos deben ser positivos, ya que suponen una utilización y valoración de los montes y permiten superar el estado de abandono anterior que, sin duda, es el que provoca mayor número de riesgos y problemas, tanto socioeconómicos como naturales.

Se hace preciso, por tanto un cambio de mentalidades acerca de los usos públicos de los ecosistemas forestales que favorecezca además la internalización de las externalidades positivas generadas por los montes mediterráneos desde el punto de vista turístico y paisajístico, para que la valoración, además de social, sea económica y, por tanto, rentable para las comarcas afectadas. Actualmente la situación exhibe un balance negativo para éstas, ya que los costes (económicos y ambientales) generados por la frecuentación turística de poblaciones alóctonas pueden ser importantes frente a unos escasos o inexistentes retornos económicos a las sociedades y espacios afectados (Eurofor, 1998: 46).

Una actitud realista, nos obliga a reconocer que en la Comunidad Valenciana los espacios forestales han sido, hasta la fecha, marginales en los planteamientos de la política territorial. Así queda patente, por ejemplo, en el documento elaborado por la Consellería de Obras Públicas sobre la formulación de un Modelo Territorial de Futuro para la Comunidad Valenciana. En el mismo se presta escasa atención a los espacios rurales del interior de la Comunidad frente a la franja litoral turística y urbanizada, no sólo en el capítulo dedicado a diagnóstico, sino también y especialmente en materia de propuestas y planteamiento de un futuro Modelo Territorial.

Por otra parte, el enfoque que caracteriza en la región al desarrollo de la política turística en espacios rurales (Vera et al., 1995) dificulta la inclusión de los aspectos ambientales y, particularmente, de los ecosistemas forestales entre sus centros de atención, a pesar de que el paisaje es uno de los principales atractivos que generan la demanda de las actividades turísticas (Yepes, 1995; Bote, 1995). No obstante, si desde el sector turístico se plantea la necesidad de cambiar el modelo de fuerte concentración espacial en la franja litoral propio de la región mediterránea y la posibilidad de utilizar el turismo como factor de reequilibrio

5 Segundo Inventario Forestal Nacional (1986-1995). Provincia de Alicante y Provincia de Valencia, p. 16. 
territorial y socioeconómico (Vera et al., 1995 y 2000), también desde las políticas de desarrollo local y desde la propia estrategia forestal se contempla el turismo como posibilidad para garantizar la gestión de los montes y del paisaje (Rojas, 1999).

Hasta la fecha no ha existido un compromiso real de interacción entre la política forestal y la política de desarrollo del turismo, entre los espacios y comarcas forestales y la oferta de recursos y servicios turísticos. Pero la Carta Europea del Turismo Sostenible en los Espacios Protegidos (2000) ofrece un marco de conexión y colaboración que debiera aprovecharse para favorecer la complementariedad y obtener el máximo rendimiento de las iniciativas planteadas desde ambos sectores en la misma dirección. La sostenibilidad del nuevo modelo de desarrollo turístico, basado en la cualificación y diversificación de la oferta, está ya siendo espontáneamente interiorizada por los agentes locales y aplicada a sus actividades desde los conceptos de la diversificación económica y la revalorización del potencial endógeno. Se trata, por tanto, de favorecer la inserción del sector y de los espacios rurales en estas redes y estrategias de desarrollo rural, en la búsqueda de un beneficio mutuo.

\section{Clasificación de los usos y funciones turístico-recreativas del monte mediterráneo}

La importancia que han ido adquiriendo las funciones turístico-recreativas de los montes mediterráneos se manifiesta en el propio hecho de que este concepto, sustituido en ocasiones por la expresión «usos públicos», está desplazando y concretando progresivamente la alusión genérica a las funciones sociales del monte. Los nuevos usos recreativos de los ecosistemas forestales mediterráneos y, en general, la actividad turística en la naturaleza, reciben actualmente una valoración social positiva por su interacción con los objetivos del desarrollo rural. Por otra parte, el aumento de la actividad recreativa en los ecosistemas forestales de la Comunidad Valenciana se estima en un 13\% anual (Cardells, 1997), lo que demuestra la importancia de estas demandas.

Una primera distinción en las funciones turístico-recreativas que desempeñan los espacios forestales nos obliga a diferenciar el uso directo del monte con fines lúdicos, de carácter intensivo y localizado en áreas recreativas, espontáneo, libre, generalmente gratuito y con estancias de una sola jornada; del uso indirecto que realizan los transeúntes ocasionales de las comarcas forestales de unos productos ambientales y paisajísticos difíciles de cuantificar, en ocasiones con estancias superiores a la jornada y pernotación en la zona. En este segundo caso, se trata de usos turísticos que generan una actividad económica de carácter regulado, preferentemente vinculada a la inciativa privada.

\section{1. Áreas recreativas}

Las zonas de uso público en los montes valencianos suelen caracterizarse por su alto valor paisajístico y por estar asociadas a la existencia de puntos de agua o pinares de repoblación. Los factores que más suelen influir en el interés o atracción que generan los «espacios naturales» de la Comunidad Valenciana son la estética del paisaje, el «reclamo del agua», la proximidad de hitos monumentales, la existencia de tradiciones populares (romerías) y la oferta de actividades deportivas y/o cinegéticas.

En general, los usos públicos están fuertemente condicionados por la existencia de áreas recreativas, por las pendientes y por la accesibilidad, que actúan como principales condicionantes de las prácticas turísticas y recreativas en los parajes montañosos de la región. De manera que el incremento de la demanda turística y recreativa en los últimos 
años no se ha traducido en una extensión espacial de las áreas de uso público de estos ecosistemas, salvo en los casos en que haya existido iniciativas públicas o privadas en tal sentido. Por lo general, lo que ha acontecido es una intensificación del uso público en áreas ya dedicadas a esta función. Las áreas recreativas creadas por la Consellería de Medio Ambiente han sido, por esta razón, los verdaderos catalizadores de la demanda. No obstante, sus características no son homogéneas, habiendo sido diferenciados las siguientes tipos (Cardells, 1997):

- Áreas recreativas, propiamente dichas, definidas como zonas acondicionadas para su utilización en estancia de día para actividades al aire libre y equipadas con servicios relacionados con el ocio y la educación ambiental, con una capacidad de acogida estimada en 50 personas.

- Zona de acampada autorizada, para una estancia temporal en tiendas de campaña y equipadas igualmente de instalaciones de ocio, educación ambiental y otros servicios. Disponen de capacidad para acoger a 60 personas.

- Campamento-acampada educativo social, destinado al uso continuado durante varios días por asociaciones juveniles para el desarrollo de actividades educativas al aire libre.

- Aulas de la Naturaleza, que promueven actividades de educación ambiental y de acercamiento a la naturaleza, y permiten el alojamiento para estancias cortas de grupos reducidos.

- Puntos de información, ubicados en el acceso a un área natural, donde se realiza un control de los visitantes y se les facilita información.

Considerando este conjunto de instalaciones recreativas, que sumaban en 1997 la cifra de 244, y según el estudio citado, la ratio superficie forestal arbolada de la región/número de instalaciones recreativas alcanzaba la cifra de 1.672 hectáreas por instalación, frente a las 5.089 de la media nacional, lo que evidencia una densidad a priori excesiva y constituye un factor de dificultad a su gestión. A ello se añaden las limitaciones presupuestarias y una localización no suficientemente planificada que plantea problemas de dispersión geográfica y no facilitan las tareas de mantenimiento. Las consecuencias suelen traducirse en problemas de deteriorio y abandono de estas instalaciones, dirigidas, por otra parte, a un tipo de población de procedencia preferentemente urbana y elevadas exigencias en servicios.

Estas circunstancias, añadidas al fuerte incremento anual de la demanda, obligan a definir una planificación regional de estas instalaciones, partiendo del principio de concertación e involucrando en la misma a las administraciones y sociedades locales y comarcales. Dicha planificación debiera considerar entre sus objetivos la diversificación de la oferta recreativa, ya iniciada por la Consellería de Medio Ambiente, el fomento de la iniciativa privada y la racionalización de la distribución geográfica de las áreas recreativas, acercándolas a las zonas con mayor densidad urbana y favoreciendo su instalación en la periferia de los núcleos de población rurales, no sólo para facilitar su mantenimiento, sino también para dar respuesta a las demandas de la población local en las comarcas del interior.

Además de las áreas de picnic, que actualmente representan el mayor porcentaje de esta oferta, conviene desarrollar la creación de centros de acogida y orientación, localizados en puntos estratégicos, que ofrezcan información sobre los valores a observar y conservar en la zona. Estos lugares pueden ofrecer además información sobre otros puntos de la comarca y actuar como centros de introducción científica y artística a partir de la exposición de imá- 
genes, textos y objetos relacionados con la historia natural y social del monte, que muestren la evolución de los ecosistemas forestales con ejemplos testimoniales. Asociados a estos centros, cabría presentar igualmente una oferta de senderismo «informado» que, aprovechando la demanda de actividades deportivas en contacto con la naturaleza, fomentara el conocimiento y la valoración del medio.

\subsection{Turismo rural}

Aunque no existe una definición del «turismo rural» generalmente admitida (Blanquer, 2000), y a pesar de la amplia gama de términos que se manejan para referirse al mismo con diferentes matices ${ }^{6}$, podría aceptarse como referencia la definición aprobada en el Congreso Mundial sobre Ecoturismo (Belize, 1992), que ha sido reproducida en diversas publicaciones: "Turismo dedicado al disfrute de la naturaleza de forma activa, con el objetivo de conocer e interpretar los valores naturales y culturales existentes en estrecha interacción e integración con las comunidades locales y con un mínimo impacto sobre los recursos, sobre la base de apoyar los esfuerzos dedicados a la preservación y utilización de las áreas naturales donde se desarrolla, o de aquellas prioritarias para el mantenimiento de la biodiversidad» ${ }^{7}$.

En la Comunidad Valenciana, el desarrollo turístico rural fue impulsado a principios de los años noventa por el antiguo Instituto Turístico de Valencia (ITVA), dependiente de la Generalitat Valenciana, y por la Unión Europea a través de iniciativas como el LEADER. Su objetivo era, según el Programa de Turismo Interior de la Generalitat Valenciana, «diversificar la oferta turística valenciana mediante la creación de un producto de Turismo Interior basado en una red de alojamiento de titularidad pública y gestión privada, en determinadas zonas con posibilidades de desarrollo turístico. Las acciones persiguen ser el germen de futuras actuaciones de iniciativa privada que potencien y consoliden dichas zonas desde la vertiente turística» ${ }^{8}$.

Pero las actuaciones realizadas se han centrado excesivamente en la creación y cualificación del alojamiento rural. Otros factores que también inciden en el desarrollo del producto, como la gestión territorial o la cualificación de la mano de obra, no han recibido una atención suficiente (Solsona, 1999: 33). La oferta de alojamiento ha sido, de hecho, el parámetro que ha experimentado una evolución más importante. Según los datos estadísticos ofrecidos por la Agencia Valenciana de Turismo (1999 y 2000), en los últimos años se ha registrado un incremento anual de la capacidad de alojamiento por encima del $50 \%$. De hecho, la normativa existente en materia de turismo rural se ocupa exclusivamente de la reglamentación del alojamiento ${ }^{9}$, al igual que suele ocurrir en otras comunidades autónomas (Blanquer, 2000).

Como reacción a este problema, el turismo rural se ha entendido en algunos casos con una fuerte carga deportiva, derivada de la «necesidad» de planificar y ofrecer actividades para entretener a los turistas. En cambio, han recibido menor atención las ofer-

6 Agroturismo, turismo verde, turismo de naturaleza, turismo de interior, turismo de pueblo, ecoturismo, turismo sostenible, turismo en casas rurales.

7 Marchena (1993), cit. en Fundació Medioambiental, 1996.

8 Cit. en Valdés, L. y Valle, E, 2000.

9 Decreto 253/1994, de 7 de diciembre, del Gobierno Valenciano regulador del alojamiento turístico rural en el interior de la Comunidad Valenciana. 
tas orientadas al «descubrimiento» de la naturaleza, cultura, tradiciones y gastronomía del lugar, que actualmente están adquiriendo un interés creciente. Es muy reciente la planificación de diferentes fórmulas de excursiones y escuelas-taller que potencian la singularidad de la oferta específica comarcal o municipal frente a la estandarización o banalización del turismo rural. En esta línea, la recuperación y el mantenimiento de los ecosistemas forestales ofrece grandes posibilidades para la planificación de actividades complementarias.

El notable incremento de la demanda que ha experimentado el turismo rural durante los últimos años en la Comunidad Valenciana ${ }^{10}$, supone que los usos y funciones turísticorecreativas de los ecosistemas forestales se han convertido en un valioso recurso potencial para muchas comarcas valencianas de interior. Sin embargo, el desarrollo de las nuevas formas del turismo rural no ha sido geográficamente homogéneo. Se ha producido una especial concentración en las de las comarcas béticas septentrionales (Els Ports-Maestrat, Alto Palancia) y en la Montaña de Alicante (L'Alcoià-Comtat y parte interior de Marina Alta y Marina Baixa), frente a unas comarcas centrales de oferta reducida (Los Serranos, Rincón de Ademuz) (Vera et al., 1995 y Cruz, 1997).

Por otra parte, tampoco sus manifestaciones son las mismas en toda la región. Al contrario, éstas acentúan las diferencias comarcales y ayudan a reconocer los diferentes intereses y potencialidades territoriales existentes en la Comunidad Valenciana. Hay que recordar, en este sentido, que la actividad turística rural no ha de ser necesariamente complemento o alternativa de la oferta dominante de sol y playa, sino que puede perfectamente ser un desarrollo turístico diferencial del existente en la costa (Solsona, 1999: 18). La zonificación de usos turísticos-recreativos que planteo más adelante insiste en la necesidad de este tratamiento diferenciado según las características territoriales y el comportamiento de la demanda. En palabras de Bote (1992), el reto consiste en «investigar en cada caso fórmulas que compatibilicen la conservación y el desarrollo mediante un uso inteligente de los recursos naturales y socioculturales disponibles».

En definitiva, la promoción del turismo en las comarcas forestales valencianas del interior ofrece numerosas ventajas y beneficios que se pueden concretar en los siguientes aspectos:

- Diversificación, ampliación y mejora de la oferta turística de la región, basada actualmente en el modelo dominante de sol y playa concentrado en la franja litoral.

- Contribución al desarrollo local de las comarcas rurales deprimidas.

Pero, el turismo en espacios naturales no induce la sostenibilidad de manera espontánea, es preciso articular medidas de planificación concretas orientadas a la atención y canalización de la demanda (Ors, 1995). Para que el turismo rural contribuya a la recuperación y al mantenimiento del equilibrio natural y de la calidad paisajística del monte mediterráneo es necesaria la elaboración y aplicación de un Plan de Usos Públicos de los montes valencianos que diagnostique y ordene los usos y funciones turístico-recreativas atendiendo a las especificidades comarcales.

10 Según el estudio realizado en 1997 por el Centro Excursionista de Valencia, bajo la dirección de la Generalitat Valenciana, sobre el uso público de los ecosistemas forestales de la región, el 34\% de los encuestados afirmaba que iba a pasar más de una noche en la zona visitada (Cardells, 1997). 


\section{Zonificación del valor turístico-recreativo de los montes valencianos}

A pesar de la diferenciación inicial establecida entre áreas recreativas y actividades de turismo rural, la clasificación de los usos públicos existentes y potenciales de los montes mediterráneos entraña una gran complejidad. El principal objetivo de este artículo es evaluar el patrimonio forestal de la Comunidad Valenciana apto para el uso público en sus diversas acepciones y clasificar el potencial de las diferentes zonas y su capacidad de atracción y respuesta a las diferentes demandas. Para ello, se ha partido de la valoración y clasificación de la demanda de usos públicos existente hacia los ecosistemas forestales y, en segundo lugar, de las características socioeconómicas y paisajísticas de la Comunidad Valenciana. La metodología empleada concuerda con la recientemente descrita por Lacaze (2000). Partiendo de los principios que de forma prevalente deben guiar la gestión de los espacios forestales en cada zona, se ha planteado una Propuesta de Base Territorial para la Planificación de Usos Turístico-Recreativos en los Montes Valencianos situados en las comarcas del interior, aceptando como demarcación geográfica de las mismas la zonificación turística empleada por la Generalitat Valenciana (Consellería de Industria, 1989; cit. en Cruz, 1998). Las categorías territoriales resultantes han sido las siguientes (Fig. 2):

- Zona A: Área prelitorial.

- Zona B: «Bosque-retablo»

- Zona C: Zona rural interior

Conviene aclarar, no obstante, que partiendo de la desigualdad que caracteriza el sistema territorial valenciano, definido por el contraste entre una franja litoral urbanizada donde se concentran población y actividades y las zonas montañosas de las comarcas interiores, afectadas desde mediados del siglo XX por un progresivo abandono demográfico y económico, el concepto de espacio «interior», objeto de análisis, no puede reducirse a una definición administrativa. El «interior» valenciano es, en realidad, un espacio socioeconómico caracterizado por su ruralidad, por su depresión socioeconómica y por disponer de un gran potencial de desarrollo todavía escasamente utilizado (Cruz, 2000; Vera, 2000). En él se concentran la mayor parte de los ecosistemas forestales valencianos que, insertos en esta dinámica de abandono, han ido acumulando una serie de transformaciones paisajísticas, riesgos naturales y nuevas características territoriales a lo largo de los últimos cincuenta años. No pueden, por tanto, considerarse comarcas forestales de interior propiamente dichas las incluidas en la zona A, así como buena parte de la zona B definida en el mapa. Pero su incorporación, basada en la zonificación turística mencionada (Consellería de Industria, 1989) permiten subrayar los contrastes territoriales y paisajísticos en los que se basa esta propuesta de planificación de usos turístico-recreativos.

La escala de análisis y planificación empleada para elaborar esta propuesta ha sido el nivel supracomarcal, aceptando los planteamientos definidos en esta materia por diferentes documentos elaborados por la Consellería de Medio Ambiente de la Generalitat Valenciana (Cardells, 1997 y 2001) y partiendo de las Demarcaciones Territoriales Homologadas definidas en 1988 por la Consellería de Administración Pública ${ }^{11}$. De todas maneras, aunque la

11 Se han obviado los complejos problemas de comarcalización que afectan a la Comunidad Valenciana (Piqueras y Carles, 1995). Y sólo de forma secundaria se ha considerado la influencia de las mancomunidades que se aproximan a la entidad comarcal en la región y de las propuestas supramunicipales integradoras que se han defi- 


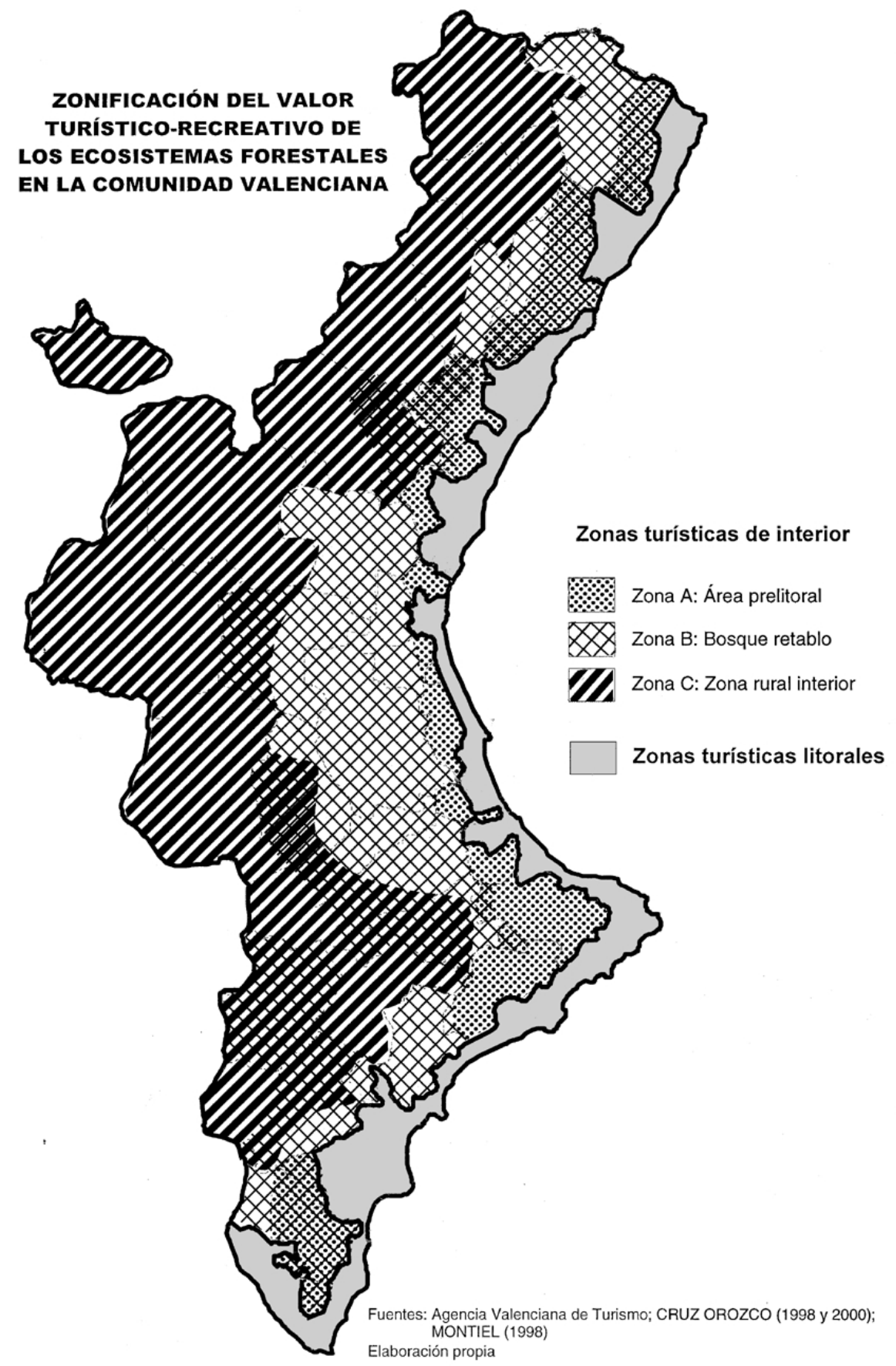

Figura 2. Zonificación del valor turístico recreativo de los ecosistemas forestales en la Comunidad Valenciana. 
escala comarcal o supracomarcal se revela como la más adecuada para la planificación territorial con carácter integrado (Vera, 2000), en muchos casos resulta más útil y expresiva la escala de análisis municipal, ya que la primera puede ocultar problemas de despoblación y desarrollo que no se manifiestan en los valores comarcales. Este es el caso, por ejemplo, de la Montaña Alicantina, donde contrasta la crisis prolongada de ciertos municipios de menor tamaño y carácter más montañoso con la terciarización y el desarrollo de los principales centros comarcales (Matarredona e Ivars, 1995: 60).

La planificación de los usos turístico-recreativos de los ecosistemas forestales debe plantearse partiendo de las potencialidades comarcales, especialmente cuando existen figuras territoriales como las mancomunidades que cuentan con redes de gestión ya desarrolladas. Pero, por supuesto, en muchos casos la aplicación de las medidas políticas habría de realizarse a escala municipal, favoreciendo la participación social.

Además del análisis y diagnóstico territorial de las zonas definidas, la propuesta incorpora una serie de posibles vías de implementación de las políticas de revalorización paisajística y uso público de los montes valencianos, diferenciando las actuaciones en función de las características y necesidades de cada zona.

\subsection{Zona A: Área prelitoral}

Esta zona se caracteriza por su proximidad a los núcleos urbano-turísticos litorales. Se trata del postpaís de la franja litoral, donde los municipios, a pesar de no contar con línea de costa, están fuertemente impregnados de la dinámica turística y urbanística del litoral, con el que mantienen un buen grado de interacción. Esta circunstancia no ejerce inicialmente una atracción principal sobre la demanda canalizada hacia el turismo rural. Por otra parte, la fuerte competencia del modelo sol/playa dominante en la región contrarresta los valores ambientales y paisajísticos de los ecosistemas forestales. Pero una política turística activa basada en la mejora de infraestructuras de comunicación y en las campañas de publicidad e información puede compensar esta desventaja inicial y transformarla en activo. Así ocurre en algunas zonas de la comarca alicantina de La Marina Alta, donde la Agencia Valenciana de Turismo (AVT) ha llevado a cabo una importante promoción turística de municipios rurales próximos al litoral y recientemente conectados a la red turística costera, destacando como ejemplo la integración y el desarrollo de Vall de Laguar (Foto 1).

El área prelitoral registra un gran número de desplazamientos y el índice de frecuentación diaria es alto, especialmente en fines de semana, puentes y períodos vacacionales. Los visitantes, procedentes de los centros urbanos de la región y de los grandes núcleos de atracción turística costeros, no suelen pernoctar en la zona (Martínez y Solsona, 2000). En suma, la demanda de usos turístico-recreativos en esta zona posee un marcado carácter urbano y niveles de formación medio-bajos en educación medioambiental. Suele ser población más exigente en servicios y comodidades que en calidad ambiental. Entre sus principales objetivos figura pasar un día de pic-nic.

El principal factor de atracción del turismo a esta zona no son los valores paisajísticos y ambientales de los ecosistemas forestales, por elevados que éstos sean, sino el carácter

nido recientemente en el marco del CONCERCOST, para el espacio de las Comarcas Centrales Valencianas (Cruz, 2000). No por ello se ha obviado la importancia territorial de estas entidades que, en ocasiones, han dispuesto de sucesivos programas LEADER que han contribuido a reforzar su cohesión y funcionalidad. Sin duda, entre los ejemplos más representativos figura el caso de Els Ports-Maestrat. 
«pintoresco» de los núcleos rurales y la proximidad y accesibilidad a los núcleos turístico-residenciales de la costa. Debiera, por tanto, extraerse el máximo beneficio de la ubicación estratégica de estos municipios, añadiendo a las motivaciones propias del turismo en espacio rural la opción de disfrutar de la oferta de ocio del litoral (Matarredona e Ivars, 1995: 75). En este caso, la incentivación del turismo rural es una estrategia complementaria que permite beneficiar a los espacios rurales costeros de los atractivos del litoral y favorecer la conservación y gestión de los montes por sus valores paisajísticos y por la complementariedad económica que representan. En definitiva, se trata de invertir la lectura que habitualmente suele plantearse en las estrategias de desarrollo territorial de las áreas turísticas del litoral: ofrecer éstas como opción complementaria del «turismo de naturaleza», y no sólo los «espacios naturales» como complemento del modelo «sol y playa» dominante.

La intensidad y el carácter de la demanda en esta zona aconsejan concentrar la oferta de áreas recreativas en esta zona, próxima al litoral urbanizado. El carácter urbano y poco concienciado sobre los valores ambientales de una población que busca principalmente pasar una jornada distendida en contacto con la naturaleza, condiciona la planificación de una red de instalaciones atendiendo a los siguientes criterios:

- Atractivo paisajístico, buena accesibilidad y posición periférica respecto a las zonas forestales de mayor valor ambiental y con mayor riesgo de incendios.

- Proximidad a los núcleos urbanos locales, complementando de esta manera la oferta de parques periurbanos dirigida a las poblaciones locales y facilitando las tareas de mantenimiento de estos espacios.

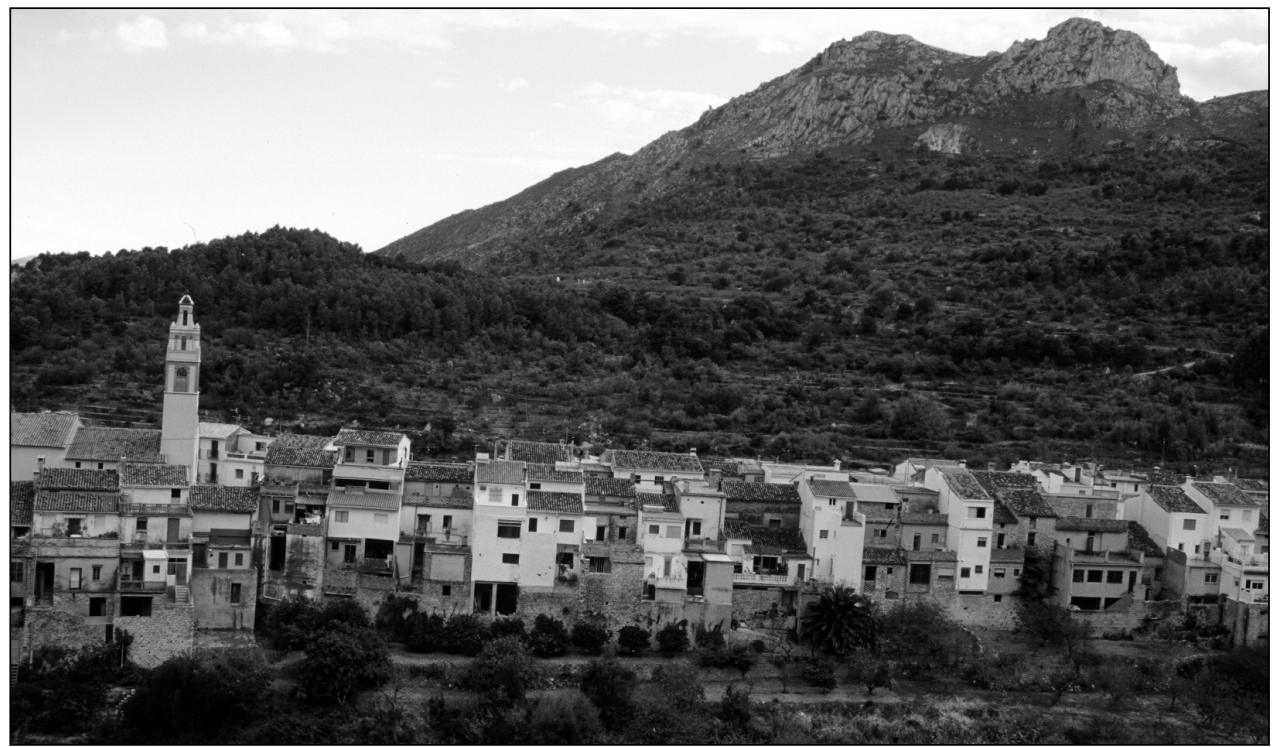

Fото 1. Revitalización de los núcleos rurales situados en la zona A, próximos a la franja litoral (Vall de Laguar, Alicante). 
Otras propuestas de gestión forestal orientada al desarrollo de las funciones turísticorecreativas en esta área son las siguentes:

- Desarrollo de los servicios vinculados a las áreas recreativas, incentivando la participación del sector privado.

- Definir circuitos de senderismo sencillos, bien señalizados y de corto recorrido que contribuyan a difundir y valorar el conocimiento de estas comarcas desde una perspectiva diferente a la propiamente litoral.

- Salvaguardar y defender los valores ambientales existentes frente a la especulación y la competencia con otros intereses y usos del suelo.

\subsection{Zona B: «Bosque-retablo»}

Es una zona de gran importancia territorial y socioeconómica en la Comunidad Valenciana, donde el vínculo que se ha establecido entre la actividad turística y el desarrollo inmobiliario, unido a la ausencia de una ordenación territorial suficiente, ha generado unas condiciones especiales que obligan a adoptar un modelo de gestión forestal adaptado a estas circunstancias.

En esta zona existen buenas comunicaciones con la franja litoral; lo cual, unido al predominio del modelo turístico «inmobiliario», genera flujos intensos de fin de semana y estivales. Por otra parte, la función paisajística que desempeñan los ecosistemas forestales como «telón de fondo» $\mathrm{y}$ «contexto ambiental» de los conjuntos de residencia secundaria, adquiere una gran importancia y llega a condicionar el valor patrimonial de las viviendas. En buena medida, el monte se convierte en un «retablo» 0 «escenario» para las urbanizaciones.

Sin embargo, los ecosistemas forestales muestran en esta zona un mayor deterioro paisajístico, en buena medida como consecuencia de reiterados incendios forestales, y son objeto de un menor conocimiento y valoración por parte de la población local. Por otra parte, predomina la propiedad privada y el minifundismo en unos espacios forestales que suelen presentar un carácter residual frente a la expansión de otros usos a lo largo del tiempo. Además, el turismo rural en esta zona es principalmente de carácter pasivo y su valoración del paisaje tiene una componente fundamentalmente estética (Foto 2).

Las propuestas de gestión forestal de los usos turístico-recreativos planteadas para esta zona son las siguientes:

- Desarrollar nuevas fórmulas de usos e instalaciones recreativas, basadas en la especialización y aprovechando el incremento de la demanda excursionista y de actividades de interpretación de la naturaleza. Entre los objetivos prioritarios cabría considerar el control y la mejora de los servicios existentes; favorecer la vertiente interpretativa, potenciando la función de estas áreas como medios de información y formación medioambiental; e incentivar la iniciativa privada para atender las crecientes demandas de origen urbano.

- Las características forestales de la zona convierten a ésta en objetivo de gestión preferente, no sólo por su valor potencial sino, sobre todo, por los problemas de degradación que presenta y por la especial concentración del riesgo de incendios forestales. La gestión de los espacios forestales obliga, por tanto, a extremar las medidas de protección en materia de prevención del riesgo de incendios y a priorizar la conservación y el mantenimiento del paisaje. 
- Entre los objetivos principales de la política forestal en esta zona debieran figurar el fomento de la asimilación de los valores ambientales por parte de la población local y visitante y la internalización de las externalidades positivas generadas por los espacios forestales.

\subsection{Zona C: Rural interior}

Las comarcas clasificadas como zona $C$ o rural interior son las peor comunicadas del interior montañoso. Han sido definidas por algún autor como «rural profundas» (Matarredona e Ivars, 1995: 71) y se caracterizan por su mala accesibilidad, por su mayor altitud y por sus pendientes. Durante los últimos quince años han sido objeto de aplicación de diferentes políticas e iniciativas de desarrollo rural. Actualmente son escenario de nuevas formas de turismo (turismo de naturaleza, ecoturismo...) en plena fase de desarrollo y que conjugan diferentes atractivos paisajísticos y ambientales, así como la oferta de actividades deportivas.

El monte representa uno de los principales valores patrimoniales en los municipios de esta zona (Domingo, 1997; Rodrigo, 1999). De hecho, presentan recursos forestales más abundantes y evolucionados que otras comarcas como consecuencia del abandono de aprovechamientos que experimentaron durante la etapa anterior a la fase actual de terciarización y de una menor presencia de los incendios forestales. Los procesos de regeneración espontánea de la vegetación han permitido, en muchos casos, la recuperación de las formaciones forestales y la aparición de comunidades vegetales evolucionadas (Foto 3).

En algunos parajes la ordenación de los usos recreativos cuenta con una larga tradición, debido a la simbología histórica, paisajística y cultural que entrañan. Es el caso, entre otros, del «Carrascal de la Font Roja» (Alcoy). Sin embargo, junto a las actividades de tipo recreativo, excursionista, religioso y deportivo que tradicionalmente se desarrollan en estos lugares, no se ha producido un desarrollo paralelo de iniciativas vinculadas a las nuevas

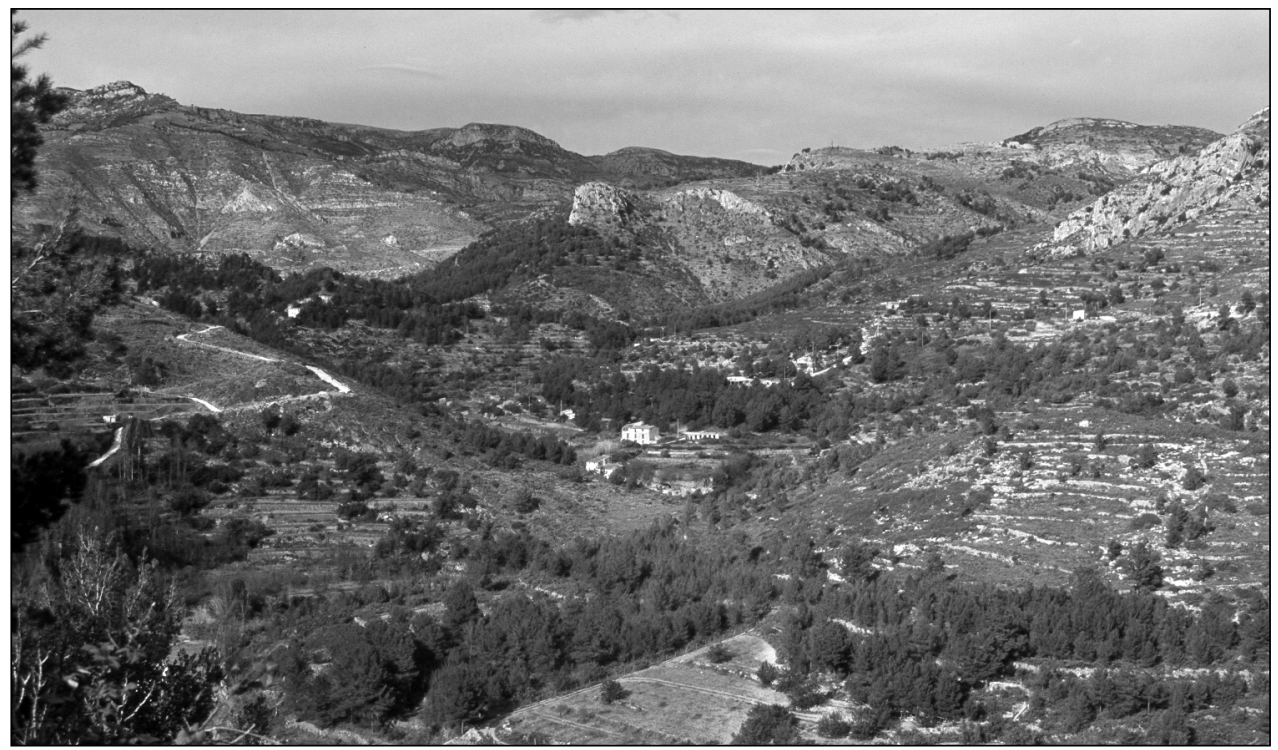

Fото 2. Minifundismo e infragestión de los ecosistemas forestales en la zona B (L’Alcalatén, Castellón). 
fórmulas del turismo rural que sí cuentan, en cambio, con cierto interés en otros municipios de la comarca o en otros lugares de la Comunidad Valenciana, como Els Ports-Maestrat. No existe, por tanto, un modelo territorial general que permita definir la situación de las comarcas valencianas del interior en relación a las actividades turístico-recreativas. Por el contrario, se aprecia una marcada heterogeneidad y contrastes que obligan a plantear una planificación del turismo rural y de los usos públicos de los espacios forestales adaptada a cada conjunto comarcal.

En cualquier caso, en esta zona se han multiplicado las iniciativas privadas orientadas a acoger turismo de estancias de larga duración, cuyo índice de frecuentación es medio-bajo. Se trata de una demanda cualificada desde el punto de vista formativo y que valora la entrada en la «intimidad de la comarca», lo que justifica la organización y oferta de un servicio de animación rural.

En consecuencia, las propuestas de gestión forestal orientada al desarrollo de los usos turístico-recreativos del monte en esta zona, son las siguientes

- Definir y clasificar los ecosistemas forestales de mayor interés y orientar su gestión, salvaguardando y contribuyendo a los avanzados procesos de regeneración de las comunidades vegetales y fomentando el conocimiento y la valoración de estos ecosistemas.

- Favorecer el contacto con una naturaleza lo menos alterada posible, practicando una gestión conservacionista y desde un modelo extensivo netamente diferenciado de las características y el modo de vida urbano.

- Definir fórmulas tendentes a la internalización de las externalidades positivas forestales (sociales y ambientales), como ya se realiza en otros ámbitos territoriales como el europeo o el norteamericano (Antón y López, 1996). El propio turismo puede aportar parte de los recursos financieros necesarios para la protección del medio y convertirse, de esta manera, en una de las vías de implementación de las políticas de revalorización paisajística y uso público de los montes valencianos.

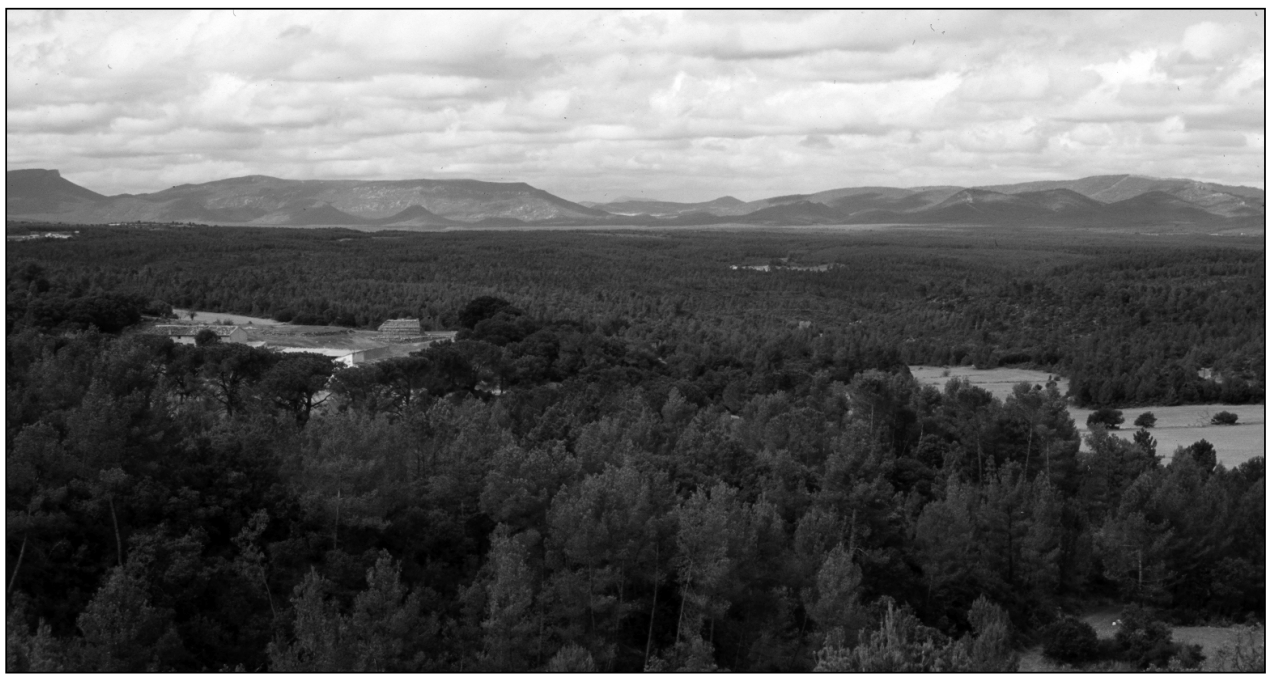

Fото 3. Regeneración y extensión de los ecosistemas forestales en la zona C (Los Serranos, Valencia). 
- Contribuir a la corrección de los procesos de despoblamiento y abandono ofreciendo alternativas de usos y empleos en el sector forestal vinculadas a los procesos de terciarización.

- Aumentar la dotación de áreas recreativas próximas a los núcleos de población, destinadas con carácter preferente a las necesidades de la población local, favoreciendo la participación municipal en la creación y mantenimiento de estos espacios.

\section{Conclusiones}

La Comunidad Valenciana se ha caracterizado hasta la fecha por una evidente descoordinación entre la política turística y las demás políticas que afectan al territorio y al medioambiente. El planteamiento sectorial que ha marcado el desarrollo del turismo en espacios rurales ha dificultado la integración de los aspectos medioambientales -y particularmente de los ecosistemas forestales - entre sus centros de atención, a pesar de que el paisaje es uno de los principales atractivos que generan la demanda de estas actividades. En general, se reconoce y subraya la importancia de los valores ambientales y paisajísticos para el desarrollo sostenible del turismo, pero no se diseñan, en paralelo, líneas políticas e instrumentos de gestión para la consecución de estos objetivos.

Las alusiones al principio de sostenibilidad que debe orientar los procesos de desarrollo de los sistemas turísticos comarcales y a la necesidad de integrar en las estrategias de planificación la conservación del paisaje natural y rural son cada vez más frecuentes, pero sólo de forma excepcional se desarrolla esta idea apuntando fórmulas concretas o, al menos, posibles cauces de incorporación progresiva de tales objetivos. Es cierto que desde el sector turístico se plantea la necesidad de cambiar el modelo territorial que sustenta estas actividades y la necesidad de utilizar el turismo como factor de reequilibrio territorial y socioeconómico; y también que desde las políticas de desarrollo rural, y desde la propia política forestal, se contempla el turismo como una posibilidad para garantizar la gestión de los montes y del paisaje.

El turismo rural se perfila en los últimos años como un posible cauce de integración de los aspectos ambientales en la planificación territorial, pero desde el sector forestal aún son tímidas las reacciones a dicha demanda social, aunque el concepto tradicional de los usos recreativos de los ecosistemas forestales con una carga marginal y peyorativa haya quedado ya obsoleto. Por otra parte, identificar la compleja función social de los montes mediterráneos con la mera adecuación de instalaciones recreativas es un error evidente que excluye el verdadero valor turístico y paisajístico de estos ecosistemas y la demanda social real. El uso público de los montes mediterráneos ya no puede enfocarse desde los planteamientos reduccionistas de impactos ecológicos provocados por un aprovechamiento intensivo. En el paisaje reside uno de los principales activos de los ecosistemas forestales mediterráneos. Por tanto, interesa fomentar la oferta turística y recreativa dirigida a la población local y foránea, de tal forma que la conservación y mejora de estos ecosistemas se convierta en uno de los principales objetivos de su gestión.

Partiendo del hecho de que el abandono de los montes por su baja rentabilidad causa unos perjuicios globales mucho mayores que el pequeño coste que supone el mantenimiento de su gestión, diferentes autores han abogado por un modelo factible de internalización de las externalidades positivas generadas por los montes mediterráneos que involucre particularmente a la actividad turística. En este sentido, la Carta Europea del Turismo Sostenible en los Espacios Protegidos del año 2000 constituye un marco idóneo para la acción. La 
valoración turístico-recreativa de los montes mediterráneos debe integrarse en las estrategias de desarrollo local y rural, superando esquemas y marcos de gestión sectoriales y cerrados. Desde esta perspectiva se puede definir un modelo de planificación abierto y participativo con una base territorial y social.

En definitiva, se trata de plantear una política de desarrollo rural sostenible y multifuncional con el monte, y no a pesar del monte (por la existencia de valores protegidos que no son asimilados, integrados ni rentabilizados en el ámbito local), contra el monte (marcada por desordenadas transformaciones urbanísticas y agrícolas y por los incendios forestales), ni al margen del monte (prolongando la etapa de abandono que ha provocado el actual riesgo de incendios y la ruina paisajística de los espacios forestales).

\section{Bibliografía}

AGENCIA VALENCIANA DE TURISMO (1999): El Turismo en la Comunidad Valenciana: 1998. Valencia.

AGENCIA VALENCIANA DE TURISMO (2000): Oferta turística municipal y comarcal: 1999. Valencia.

AIFM (2002): Problématique de la forêt méditerranéenne. Forêt Méditerranéenne, hors série, $\mathrm{n}^{\circ} 1$-août 2002. Marsella. $191 \mathrm{pp}$.

ANTON CLAVE, S. y LÓPEZ MONNE, R. (1996): «Turismo rural, desarrollo local y preservación del ambiente. Elementos para un desarrollo sostenible del turismo en la zona de Montaña Prades-Montsant, Cataluña», Ería, no 26, pp. 227-238.

BLANQUER, D. (dir.) (2000): Turismo: comercialización de productos, gestión de organizaciones, aeropuertos y protección de la naturaleza. II Congreso Universidad y Empre$s a$, Valencia, Tirant lo Blanch, pp. 486-496.

BOTE, V. (1992): «Nuevas formas de turismo para la revitalización del espacio interior en España», Rassegna di Studi Turistici. Roma, Associazione Nazionale Italiana Esperti Scientifici del Turismo, no 3-4, pp. 217-236.

BOTE, V. (1995): La demanda turística española en espacio rural o de interior: situación actual y potencial. Madrid, Instituto de Economía y Geografía (C.S.I.C.), mecanografiado.

CARDELLS, F. (dir.) (1997): Valoración económica del uso público de los ecosistemas forestales de la Comunidad Valenciana. Estudio elaborado por el Centre Excursionista de València con la colaboración de la Consellería de Medio Ambiente de la Generalitat Valenciana. Valencia. Mecanografiado.

CARDELLS, F. (2001): Valoración de los recursos ambientales y naturales. Universidad Politécnica de Valencia, Centro de Ingeniería Económica. Valencia, 302 pp.

Carta Europea del Turismo Sostenible en los Espacios Protegidos (2000), ed. Generalitat de Catalunya

CONSELLERIA DE INDUSTRIA, COMERCIO Y TURISMO (1989): Metodología de la encuesta turística (Hoteles y hostales). Valencia, Generalitat Valenciana.

CONSELLERIA DE OBRAS PUBLICAS (2000): Modelo Territorial de la Comunidad Valenciana. Generalitat Valenciana, Valencia.

CONSEJO DE EUROPA (1997): Tourism\&Environment, Questions and Answers, $\mathrm{n}^{\mathbf{o}} 3$, Strasbourg.

CRUZ OROZCO, J. (1997): «El turismo en espacios rurales en el País Valenciano», en VALENZUELA, M.: Op. cit., pp. 663-673. 
CRUZ OROZCO, J. (1998): Áreas de montaña y políticas de desarrollo rural: el turismo en el País Valenciano, tesis doctoral dirigida por J. Romero González y presentada en la Facultad de Geografía e Historia de la Universidad de Valencia, inédita.

CRUZ OROZCO, J. (2000): «El aprovechamiento de los espacios naturales del interior del País Valenciano como recurso económico», en MARTÍNEZ PUCHE, A.-PÉREZ, D.-SANCHO, I. (coords.): Herramientas para el Desarrollo Local, Universidad de Alicante, pp. 267-303.

Declaración de Québec sobre Ecoturismo, de 22 de mayo de 2002, Cumbre Mundial de Ecoturismo. Disponible en www.world-tourism.org

DOMINGO PÉREZ, C. (1997): «El interior de Castellón: el turismo como alternativa», en VALENZUELA, M.: Op. cit., pp. 379-391.

EUROFOR (1998): La forêt et l'Europe. Luxemburgo, Parlamento Europeo.

FUNDACIÓ MEDIOAMBIENTAL (1996): Els Ports-Maestrat: Una propuesta de gestión para el futuro. Fase I. Difusión ambiental: Educación ambiental y ecoturismo (extractoresumen, mayo 1996). Inédito.

LACAZE, J. (2000): «Forest management for recreation and conservation: new challenges», en Forestry. Journal of the Institute of Chartered Foresters, Oxford University Press, pp. 137-141.

MARTÍNEZ, F.J. y SOLSONA, J. (2000): Alojamiento turístico rural: gestión y comercialización, Madrid, Síntesis.

MATARREDONA, E. e IVARS, J. (1995): «El turismo rural: Una alternativa para el desarrollo integrado de los municipios rurales de la montaña alicantina», Investigaciones Geográficas, Universidad de Alicante, $\mathrm{n}^{\circ}$ 14, pp. 59-76.

MERLO, M. y ROJAS, E. (2000): «Public goods and externalities linked to Mediterranean forests: economic nature and policy», Journal of Land Use Policy, Elsevier Science Ltd, no 17, pp. 197-208.

MINISTERIO DE MEDIO AMBIENTE (2000): Estrategia Forestal Española. Organismo Autónomo de Parques Nacionales, Madrid.

MONTIEL, C. (1994): «Práctica y regulación del uso público de los espacios protegidos en la provincia de Alicante», II Congreso de Ciencia del Paisaje. Barcelona, Universitat de Barcelona-Fundació «La Caixa», vol. 2, pp. 355-367.

MONTIEL, C. (1996 y 1999): «Evolución histórica de la política forestal en la Comunidad Valenciana (I y II)» Revista Forestal Española, no 14, pp. 4-11 y nº 22, pp. 28-34.

ORS, J. (1999): «El turismo y la conservación de los espacios naturales», en VIÑALS, M.J. y BERNABÉ, A.: Op. cit., pp. 37-57.

PIQUERAS, J. y CARLES, J. (1995): «La política territorial de la Generalitat Valenciana: la comarcalització pendent», Cuadernos de Geografía, Universidad de Valencia, ${ }^{\circ} 58$, pp. 337-364.

REYNA, S. (1992): «El turismo rural como factor determinante en la conservación de los recursos naturales y socioeconómicos y en el desarrollo local», en El turismo rural en el desarrollo local. MAPA. Madrid, pp. 5-13.

RODRIGO ALONSO, C. (1999): Puebla de San Miguel: «el rincón del Rincón», Ayuntamiento de Puebla de San Miguel, 63 pp.

ROJAS, E. (1999): «Alternativas a la financiación sostenible del paisaje balear», en Actas del Seminario: La problemática del «lloc menorquí» en orden al equilibrio medioambiental, $4^{\circ}$ vol, AGRENA, Ciutadella de Menorca, pp. 179-192. 
SOLSONA, J. (1999): El turismo rural en la Comunidad Valenciana: análisis y planificación. Aplicación al Alto Mijares. Castellón de la Plana, Sociedad Castellonense de Cultura.

VALDÉS, L. y VALLE, E. (2000): «Experiencias comparadas de turismo rural en España», II Congreso Universidad y Empresa, Valencia, pp. 575-600.

VALENZUELA RUBIO, M. (coord.) (1997): Los turismos de interior: El retorno a la tradición viajera. Madrid, Ediciones de la Universidad Autónoma de Madrid.

VERA, F., CRUZ, J. y BAÑOS, C.J. (1995): «Turismo y organización del territorio: desajustes de un modelo de implantación y nuevas estrategias», Cuadernos de Geografía, Universidad de Valencia, nº 58, pp. 439-474.

VERA, F. (2000): «El modelo turístico de la Marina Alta: Apuntes para una nueva etapa desde la sostenibilidad como referencia», Investigaciones Geográficas, Alicante, Instituto Universitario de Geografía, no 24, pp. 133-141.

VIÑALS, M.J. y BERNABÉ, A. (coord.) (1999): Turismo en Espacios Naturales y Rurales, Valencia, Universidad Politécnica de Valencia.

YEPES, V. (1995) «Turismo sostenible en el interior de la Comunidad Valenciana: Posibilidad de un desarrollo turístico complementario al del <sol y playa〉», Papers de Turis$m e$, Valencia, Institut Turístic Valencià, $\mathrm{n}^{\circ}$ 17, pp. 77-87. 